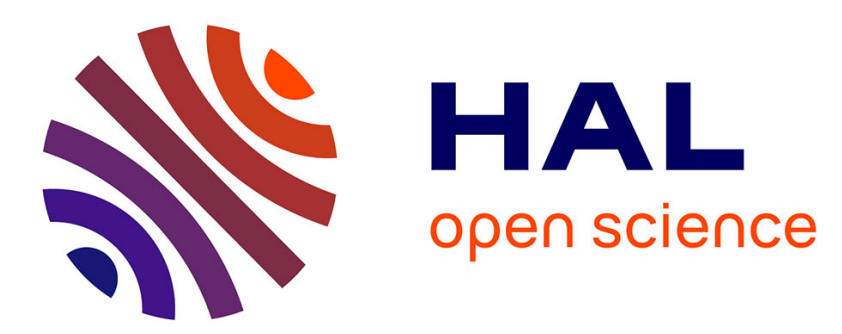

\title{
The Use of Two-Stream Approximations for the Parameterization of Solar Radiative Energy Fluxes through Vegetation
}

\author{
Joachim H. Joseph, Jean Iaquinta, Bernard Pinty
}

\section{To cite this version:}

Joachim H. Joseph, Jean Iaquinta, Bernard Pinty. The Use of Two-Stream Approximations for the Parameterization of Solar Radiative Energy Fluxes through Vegetation. Journal of Climate, 1996, 9 (10), pp.2326-2336. 10.1175/1520-0442(1996)0092.0.CO;2 . hal-02025388

\section{HAL Id: hal-02025388 \\ https://hal.uca.fr/hal-02025388}

Submitted on 9 Apr 2021

HAL is a multi-disciplinary open access archive for the deposit and dissemination of scientific research documents, whether they are published or not. The documents may come from teaching and research institutions in France or abroad, or from public or private research centers.
L'archive ouverte pluridisciplinaire HAL, est destinée au dépôt et à la diffusion de documents scientifiques de niveau recherche, publiés ou non, émanant des établissements d'enseignement et de recherche français ou étrangers, des laboratoires publics ou privés. 


\title{
The Use of Two-Stream Approximations for the Parameterization of Solar Radiative Energy Fluxes through Vegetation
}

\author{
JoAChim H. JosePh, * JeAn IAQuinta, ANd BERNARd PinTy \\ L.A.M.P.-Universite Blaise Pascal, Aubiere, France
}

(Manuscript received 15 March 1995, in final form 4 December 1995)

\begin{abstract}
Two-stream approximations have been used widely and for a long time in the field of radiative transfer through vegetation in various contexts and in the last 10 years also to model the hemispheric reflectance of vegetated surfaces in numerical models of the earth-atmosphere system.

For a plane-parallel and turbid vegetation medium, the existence of rotational invariance allows the application of a conventional two-stream approximation to the phase function, based on an expansion in Legendre Polynomials. Three conditions have to be fulfilled to make this reduction possible in the case of vegetation. The scattering function of single leaves must be bi-Lambertian, the azimuthal distribution of leaf normals must be uniform, and the azimuthally averaged Leaf Area Normal Distribution (LAND) must be either uniform or planophile. The first and second assumptions have been shown to be acceptable by other researchers and, in fact, are usually assumed explicitly or implicitly when dealing with radiative transfer through canopies. The third one, on the shape of the azimuthally averaged LAND, although investigated before, is subjected to a detailed sensitivity test in this study, using a set of synthetic LAND's as well as experimental data for 17 plant canopies.

It is shown that the radiative energy flux equations are relatively insensitive to the exact form of the LAND. The experimental Ross functions and hemispheric reflectances lie between those for the synthetic cases of planophile and erectophile LANDs. However, only the uniform and planophile LANDs lead to canopy hemispheric refiectances, which are markedly different from one another.

The analytical two-stream solutions for the either the planophile or the uniform LAND cases may be used to model the radiative fluxes through plant canopies in the solar spectral range. The choice between the two for any particular case must be made on the basis of experimental data.
\end{abstract}

\section{Introduction}

The initial motivation for simulating the radiative effects of vegetation was the need to model photosynthesis and, in general, the energy, gas, and moisture balances of vegetation. Monitoring the global distribution of vegetation using remote sensing tools and inversion techniques has recently led to a variety of physically based or empirical models for reflected radiances emerging from vegetation (e.g., Myneni et al. 1991; Dickinson et al. 1990; Pinty et al. 1990) as well as to maps of the seasonal variation of vegetation cover (Tucker 1979). The modeling capability has grown in the last 10 years to include the vertical coupling between vegetation, soil, and atmosphere in earth-atmosphere models (e.g., Sellers et al. 1986).

* Permanent affiliation: Department of Geophysics and Planetary Sciences, Tel-Aviv University, Tel-Aviv, Israel.

Corresponding author address: Prof. Joachim H. Joseph, Department of Geophysics and Planetary Sciences, Tel-Aviv University, Ramat-Aviv, Tel Aviv 69978, Israel.

E-mail: yoya@jupiter1.tau.ac.il
Two-stream approximations have been popular for description of the transfer of solar radiation through vegetation for a very long time (e.g., Kubelka and Munk 1931; Schwartzbart and Shaviv 1976; Dickinson et al. 1987), much before the first efforts to include vegetation in climate models (Gutman et al. 1984; Sellers et al. 1986; Kimes et al. 1987).

In the case of the large-scale general circulation models (GCMs) with spatial resolution of the order of hundreds of kilometers in the horizontal and perhaps fifty to a hundred millibars in the vertical direction, vegetation is modeled as part of the surface boundary conditions on hemispheric reflectance, emittance, roughness, and evapotranspiration (e.g., Avissar 1992; Dickinson et al. 1987; Sellers et al. 1986; Koster and Suarez 1992). When the vegetation canopy is resolved in the vertical direction in very highresolution earth-atmosphere models but is averaged horizontally over a grid unit, one-dimensional radiative flux profiles are required. Suitably averaged fast and accurate parameterizations of the radiative transfer problem through vegetation with full three-dimensional inhomogeneity for use in such high-resolution models of the earth-atmosphere system are not available as yet. 
The mathematical theory of radiative transfer through vegetation has been well developed (e.g., Ross 1981; Myneni et al. 1989; Knyazikhin and Marshak 1991; Knyazikhin et al. 1991). We can therefore dispense with its presentation in this paper except for making a few general points.

Speaking most generally, the radiation transfer process in the canopy takes place in a system in which the optically active entities are not small compared to the size of the system. Mathematically speaking, the vegetation canopy is therefore not a turbid medium and, in principle, conventional radiative transfer theory, including the two-stream approximation, is not applicable (e.g., Shifrin 1968; Ross 1981; Myneni et al. 1989). The use of such solutions must therefore be justified in each application or generally. This paper is an attempt to validate one of the conditions of applicability of twostream approximations.

It should be mentioned here that the condition of turbidity is different from that of the optically active elements being small compared to the wavelength of the incident radiation. The latter leads to optical behavior of the Rayleigh type, the former is independent of wavelength and leads to the possibility of neglecting the exact relative positions and orientations of the individual scattering elements (Shifrin 1968).

The vegetation canopy may be defined as composed of clumps of leaves separated by empty gaps, by branches, and by trunks. The single leaves, clumps, and branches may all shadow one another (e.g., Ross 1981; Knyazikhin et al. 1991). The architecture of the canopy is neither completely regular nor completely random at any spatial scale and varies in time on many scales. The relative positions and orientations may show organization. The orientations may exhibit a-possibly nonuniform-distribution in every canopy. The horizontally averaged probability distribution function (pdf) of the angle of orientation of a leaf with respect to the upper hemisphere at a given height, $z$, in the canopy is usually defined as

$$
g_{L}\left(z, \vec{\Omega}_{L}\right),
$$

where $z$ is depth into the canopy and $\vec{\Omega}_{L}$ is the direction of the leaf normal with respect to the zenith. The pdf is normalized with respect to the upper hemisphere,

$$
\frac{1}{2 \pi} \int_{2 \pi+} g_{L}\left(z, \vec{\Omega}_{L}\right) d \vec{\Omega}_{L} \equiv 1 .
$$

The probability density function for a specific plant is determined by measurements. Synthetic pdfs are often used (Ross 1981).

The so-called Ross Function (Ross 1981) defines the mean projection of the leaf normals of the distribution $g L$ in the direction $\vec{\Omega}$ :

$$
G(z, \vec{\Omega})=\frac{1}{2 \pi} \int_{2 \pi+} g_{L}\left(z, \vec{\Omega}_{L}\right)\left|\vec{\Omega}_{L} \cdot \vec{\Omega}\right| d \Omega_{L}
$$

This leads to the following definitions of the extinction coefficient, $\beta$,

$$
\sigma(z, \vec{\Omega})=G(z, \vec{\Omega}) u_{L}(z)
$$

where $u(z)$ is the leaf area density or the total onesided leaf area per unit volume at depth $z$. The existence of gaps makes the cross sections for one extinction event depend on the previous one. The wavelength-dependent scattering and absorption cross sections vary with the reflectivity and transmissivity of the single leaves as well as with the architecture of the canopy. The single scattering albedo of the medium is therefore different from that of a single leaf and will, in general, depend on angle of view as well as on location. In general, the extinction coefficient is a function not only of $z$ but also of direction.

The optical depth between the points $z$ and $z^{\prime}$ in the direction $\vec{\Omega}$ is defined by

$$
\delta\left(z, z^{\prime}, \vec{\Omega}\right)=\int_{z^{\prime}}^{z} \sigma(s, \vec{\Omega}) d s .
$$

Finally, the scattering phase function of a leaf with a given orientation, $\vec{\Omega}_{L}$, illuminated by light from direction $\vec{\Omega}$ is defined as

$$
\gamma_{L}\left(\vec{\Omega}_{L}, \vec{\Omega} \rightarrow \vec{\Omega}^{\prime}\right),
$$

where $\vec{\Omega}^{\prime}$ is the direction of emergence and the scattering angle is the one between the directions $\vec{\Omega}$ and $\vec{\Omega}^{\prime}$. The scattering phase function in this case is, therefore, in contrast to the conventional case, the conditional probability, namely, that a photon be scattered from one direction into the other. The condition is given by the relevant leaf area normal in each scattering act. The connection between an incident and an emerging beam of radiation in a single event of scattering from a canopy is not direct, unique, and symmetric but probabilistic in nature. This means that the equation of transfer is not rotationally invariant. It is therefore not possible, in general, to solve the transfer equation by expanding the scattering function in a series of associated Legendre Polynomials.

The applicability of two-stream approximations to the problem of radiative transfer through vegetation was nevertheless investigated by several researchers (e.g., Ross 1981; Dickinson et al. 1987; Myneni et al. 1989; Knyazikhin et al. 1991). In particular, Dickinson et al. (1987) showed that when the canopy is homogeneous and semi-infinite and the leaves scatter isotropically, two-stream approximations are accurate to about five percent. In a second paper (Dickinson et al. 1990 ) it has been demonstrated that models of canopy hemispheric reflectance for infinite optical depthwhen based in part on two-stream approximationsare very accurate in the case of three different synthetic leaf orientations: uniform, erectophile, and planophile. This was done by validating the results of a model for hemispheric reflectance-exact for single scattering 
with inclusion of multiple scattering based on a twostream approximation - with the Monte Carlo-based ray-tracing model, developed by Kimes (Kimes 1984). It was moreover shown in the Dickinson et al. paper that the hemispheric reflectances of canopies with vertically inclined and uniformly distributed isotropically scattering leaves are very similar except for near overhead sun. The hemispheric reflectance of a canopy with horizontal leaves was shown to be significantly different from the other two.

The main point of the present paper is a generalization and amplification of the above conclusions. We shall assume, in common with all previous work, that a canopy is a turbid medium so that the conventional radiative transfer equation is applicable. This however, also means, that shadow and hot spot effects may be included only through add-on parameterizations. Three more specific assumptions are then sufficient to reduce the photon transport equation for a plane-parallel turbid vegetation medium to a rotationally invariant form. The scattering function of single leaves must be bi-Lambertian (Myneni et al. 1988), the azimuthal distribution of leaf normals must be uniform. Third, the LANDs must be either uniform (Myneni et al. 1989) or planophile (Ross 1981).

The assumption that the LAND is uniform in azimuth has been shown to be acceptable for modeling radiative transfer through vegetation with closed canopies by several authors (e.g., Ross 1981 and references therein).

The special case of heliotropism may be modeled by a combination of a delta-function approximation for the pdf of the leaf zenith angle multiplied by a function of the cosine of the difference of the leaf and incidence azimuth angles (Verstraete 1987). For this case a twostream approximation may be developed (Ross 1981), which will also lie between the two extremes cited previously.

The bi-Lambertian scattering model for a leaf was first suggested by Ross and Nilson, (1968) and then extensively tested (Gutschick and Wiegel 1984). It basically means that both sides of the leaf scatter isotropically but with different magnitudes. It has been extensively applied for use in analyzing remotely sensed data on vegetation properties (e.g., Myneni et al. 1988; Myneni et al. 1989).

The question remaining now is how well irradiance (radiation fluxes) through vegetation can be modeled by using one of only two LANDs-uniform or planophile.

Therefore, the sensitivity of the flux RTE to the form of the LAND is a central and crucial part in the application of two-stream approximations to flux radiative transfer through vegetation and is the main focus of this paper.

In section 2 we compare theoretical and experimental Ross functions. Then we present bidirectional reflection functions (BRFs) and hemispherical reflec- tances for three theoretical LANDs for a variety of conditions. Section 3 summarizes our results and their implications.

\section{Comparison of hemispheric reflectances for different LANDs}

We show in this section that use of very different LANDs to calculate hemispheric reflectances in several radiation transfer model results in only two sets of irradiances-radiation fluxes - which are considerably different one from the other. One set is for the planophile LAND, the second includes the other LANDs. We have made a set of calculations of Ross functions, bidirectional reflection functions, BRFs weighted by the cosine of the zenith angle, and hemispheric reflectances with the different LANDs.

In Fig. 1 we show the three theoretical Ross functions, $G(z, \vec{\Omega})$, (Myneni et al. 1989) derived from the leaf area normal distributions (LANDs) that we have used and that cover the possible range of variation.

The shape of the Ross function essentially determines - given the leaf area index and the single leaf optical properties - the extinction law of a given type of vegetation. The erectophile, planophile, plagiophile, extremophile, and uniform LANDs have been suggested as being representative of natural vegetation (Bunnik 1978). The Bunnik planophile function has the property that $50 \%$ of the leaves have normals within about $20^{\circ}$ of the zenith. The same is true for the erectophile distribution, but for the horizon direction. Plants with a uniform LAND have no preferred direction of leaves. The two other distributions describe leaves mostly at $45^{\circ}$ elevation, plagiophile, and mostly erect and planar in orientation, extremophile. We do not show the results and conclusions for the latter two for reasons of clarity. All five of these have been used by many researchers to describe the optical behavior of plants.

For comparison to the theoretical functions, we also show in the same figure the experimental Ross functions calculated from measurements of 17 different plants. These functions are based on numerical integration, using experimental LANDs. Most lie completely between the planophile and erectophile models. Twelve of the particular set of plants shown have a planophiletype variation with zenith angle of incidence. The variation of the Ross function with angle of the remainder lies close to or between the uniform and erectophile cases. The first group might be parameterized by the planophile LAND and the second by either the uniform or the erectophile cases. We shall confirm further on in more general terms than previously (Dickinson et al. 1990 ) that the hemispheric reflectances of plants with uniform and erectophile LANDs differ very little one from the other. Possibly, therefore, for the purpose of modeling irradiances, vegetation may be divided into two groups. It is serendipitously fortunate that the ex- 

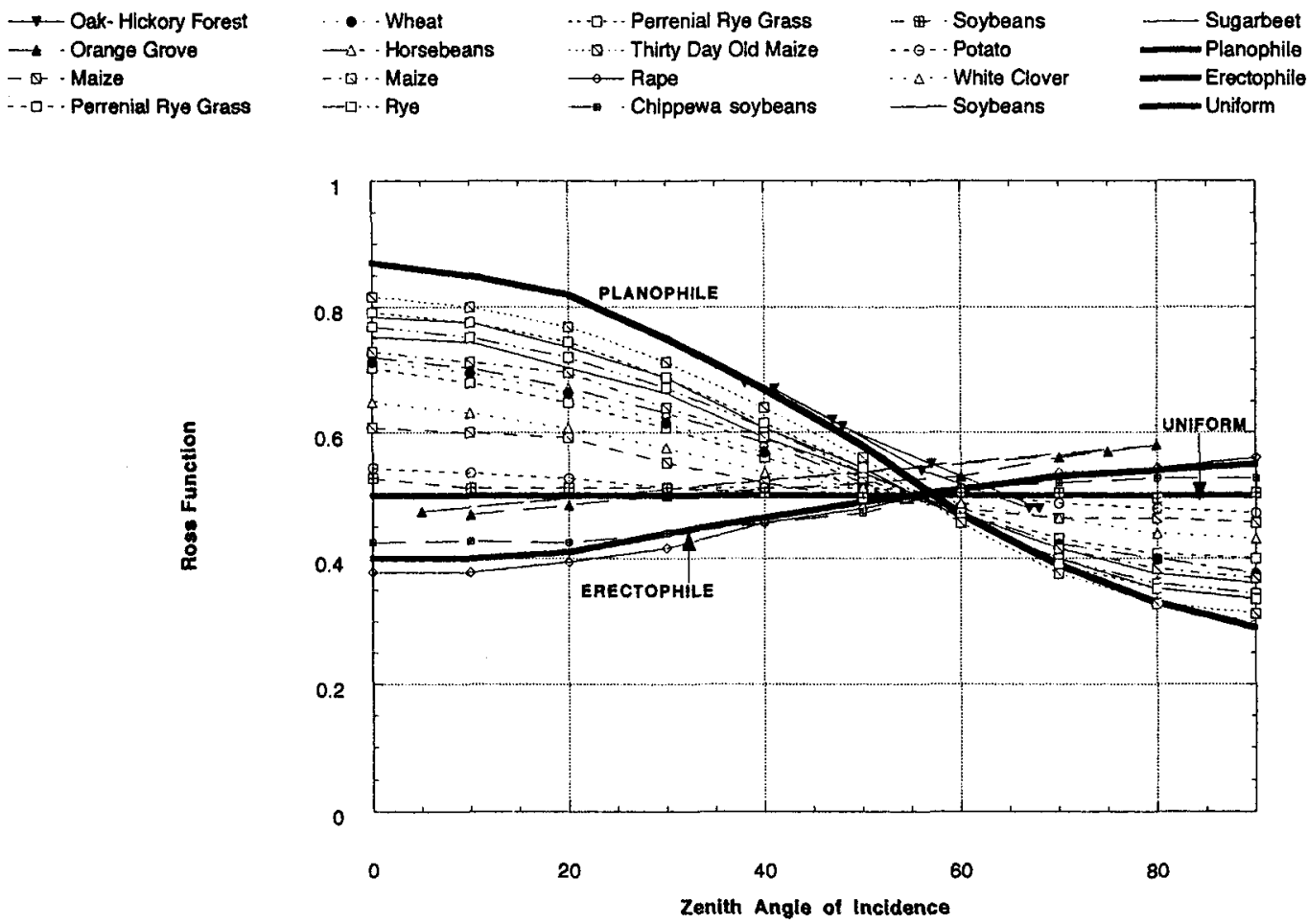

FIG. 1. Theoretical and experimental Ross functions used in this study. Oak-Hickory Forest (Baldocchi et al. 1985), Orange Grove (Cohen and Fuchs 1987), all other plants (Myneni et al. 1988 and references cited therein). The theoretical functions are based on the following LANDs (Bunnik 1978). Uniform: $2 / \pi$; Planophile: $(2 / \pi)\left[1+\cos \left(2 \Theta_{L}\right)\right]$; Erectophile: $(2 / \pi)\left[1-\cos \left(2 \Theta_{L}\right)\right]$.

istence of either a uniform or a planophile leaf angle normal distribution allows analytical two-stream approximation solution to the radiative transfer equation (Ross 1981; Myneni et al. 1989). To carry out the division of vegetation into two groups in practice, a much more extensive set of experimental data than shown in Fig. 1 must be at hand. In preparation for the availability of such a database, we use three synthetic LANDs to calculate BRFs. We then analyze here the differences between the BRFs, radiances and irradiances of vegetation for each of three LANDs - planophile, erectophile, and uniform. The calculations of BRFs are made twice, first with a new ID discrete ordinate code developed by two of us (Iaquinta and Pinty 1994) and then validated with a fully threedimensional discrete ordinate code (Myneni et al. 1992). Leaf area indices (LAIs) cover a range from one to eight - from thin to thick canopies. Surface hemispheric reflectances go from normal to very high in both the PAR -0.075 to 0.20 - and the near-IR band (NIR) -0.15 to 0.35 , respectively. Solar zenith angles cover $5^{\circ}-80^{\circ}$.

In Fig. 2a, we show perspective views of the threedimensional surfaces produced by BRFs on a polar plot in the zenith and azimuth angles. The zenith angle is the radial coordinate so that the zenith is in the center of the polar plot and the zenith angle increases from $0^{\circ}$ to $90^{\circ}$ in the radial direction. The azimuth angle increases counterclockwise from the "back" of the figure. The square base, shown in perspective, serves only to set off the 3D BRF surface from its surroundings. On the top left-hand side of Fig. $2 \mathrm{a}$, is a $3 \mathrm{D}$ perspective view of the BRF of the uniform case and underneath it similar perspective views of the difference between either the planophile or the erectophile BRFs from the uniform one. The LAI was chosen to be 8 , in order to show the case of a dense canopy. The spectral region for this figure is the near-IR band, the leaf reflectance (RL) is 0.44 , and its transmittance (TL) is 0.51 . The hemispheric reflectance of the underlying surface is 0.15 , corresponding to a typical value for vegetated soil surfaces. The solar zenith angle (SZA) is $5^{\circ}$, namely, a high sun case. The maximal relative difference of the erectophile BRF from the uniform one is relatively small, of the order of $10 \%$. In absolute terms this difference is less than 0.04 . The planophile BRF deviates much more from the uniform one, with large relative differences of $25 \%$ occurring.

The upward $(+)$ or downward $(-)$ irradiance must be evaluated by performing

$$
\pi F^{ \pm}(z)=\int_{0}^{2 \pi} d \phi \int_{0}^{ \pm 1} d \mu I(z, \mu, \phi) \mu .
$$



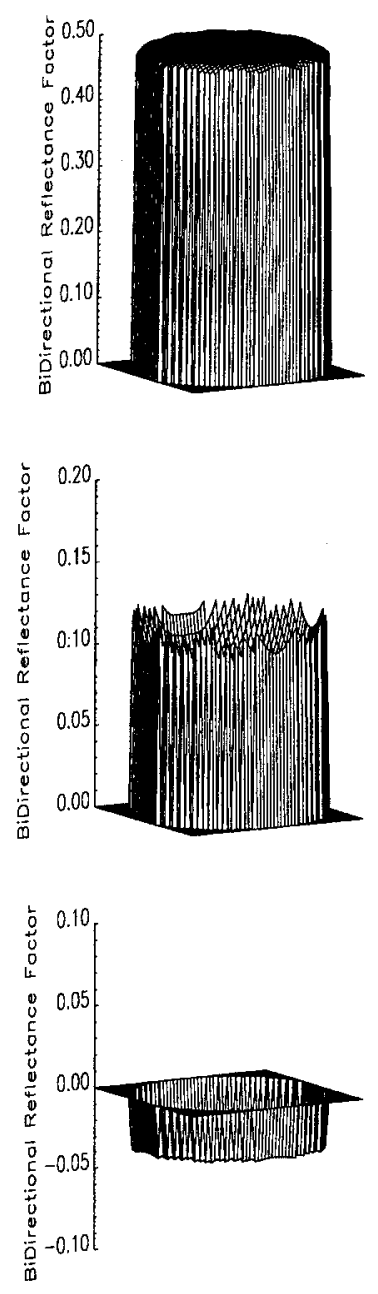
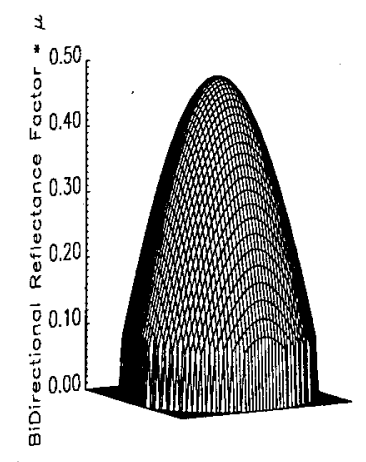

Uniform
$\theta_{0}=5$.

$\phi_{0}=180$.

$\mathrm{LAI}=8$.

$R_{L}=0.4357$

$T_{L}=0.5089$

Soil albedo $=0.15$

No hot-spot
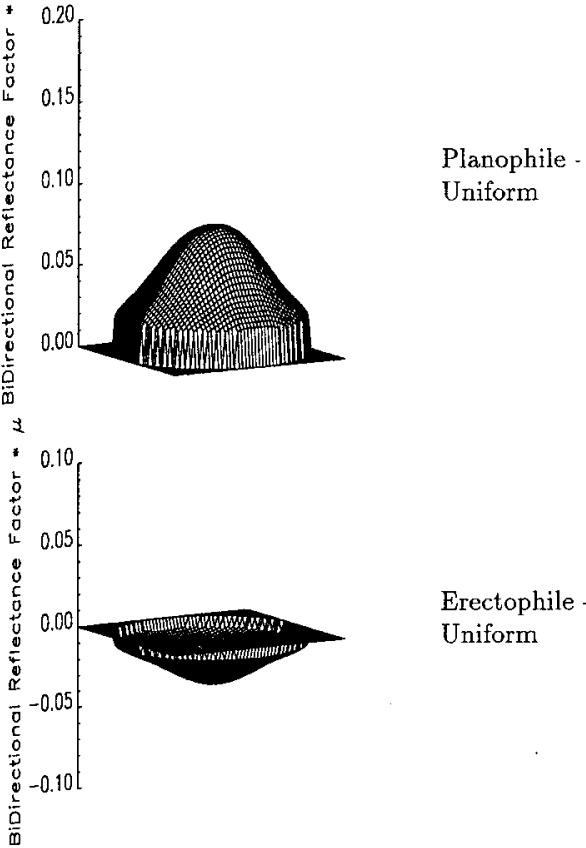

Erectophile Uniform

FIG. 2. (a) BRFs, cosine weighted BRFs and differences between planophile, erectophile, and uniform quantities for the near-IR band (NIR) at SZA $=5^{\circ}$. (b) Same as in Fig. Ia but at $S Z A=80^{\circ}$.

The radiance-BRF times amplitude factor-is weighted with the cosine of the zenith angle of emergence angle. To show the effect of weighting the radiance, $I(z, \mu, \phi)$, by the cosine of the zenith angle, $\mu$, we show on the right-hand side of the figure at the top, the uniform BRF multiplied by the cosine of the zenith angle. The high values of the BRF at all azimuth angles for directions near the horizon compared to its value near the zenith are completely suppressed and the figure shows essentially a cosine variation with a maximum at the zenith. The erectophile minus uniform surface, shown at the bottom right-hand side of the figure shows small positive and negative deviations from the uniform case. On the other hand, the difference of the weighted planophile and uniform BRF's, shown in the middle panel on the right-hand side, behaves very differently and is larger than in the erectophile case.
Figure $2 b$ shows the same information for the same case but for a large solar zenith angle of $80^{\circ}$. Even though the BRF varies strongly with viewing azimuth and zenith angles, the BRF multiplied by the cosine of the viewing angle again shows a much smoother behavior as well as a reduced magnitude. The important differences from the uniform case are again found for the planophile BRF. However, the latter differences are both negative and positive, leading - as will be shown next - to a value for the hemispheric reflectance that is not much different from that of the uniform case.

It is obvious that the large differences between the three BRFs at all azimuths-especially for the large zenith angles - are very much reduced by weighting the BRF with the cosine of the zenith angle.

Let us now analyze the upward irradiances as a function of the cosine of the solar zenith angle for two LAIs and the three LANDs. The first summary we would like 

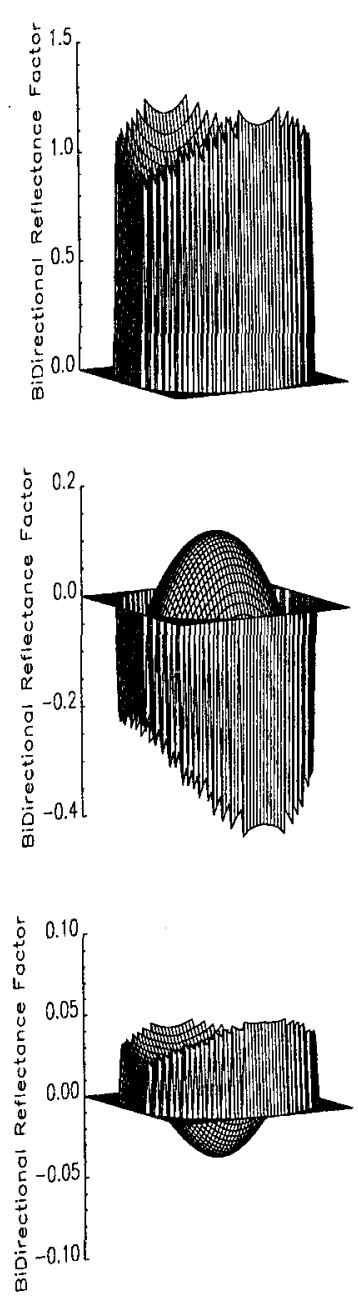

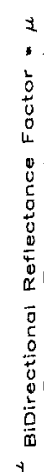

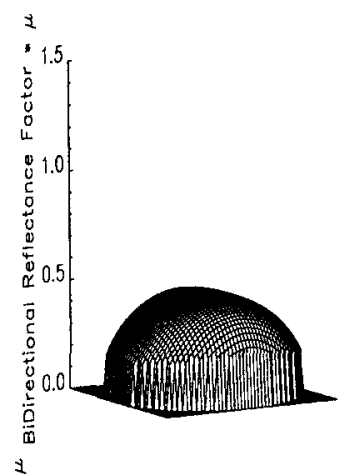

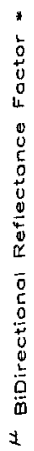
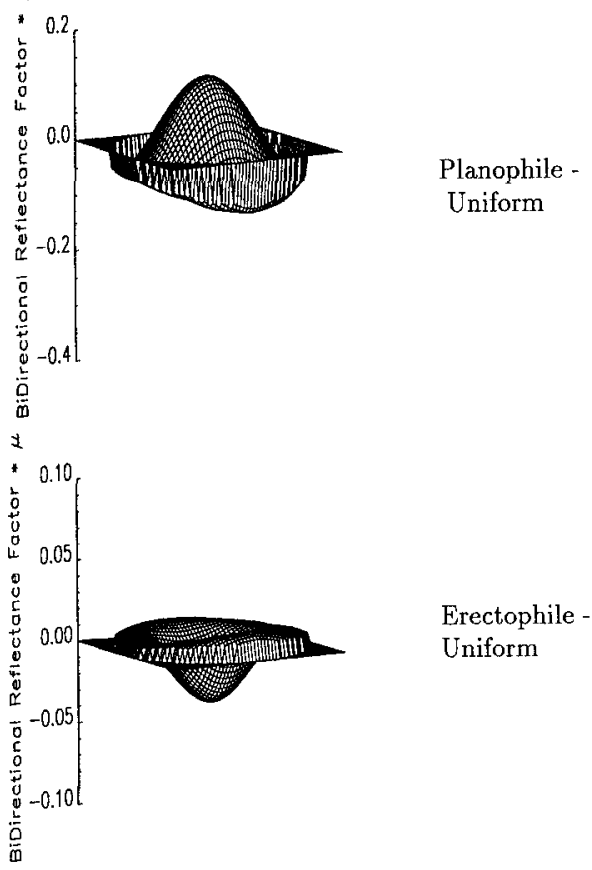

FIG. 2. (Continued)
Uniform

$\phi_{0}=180$.

$\mathrm{LAI}=8$.

$R_{L}=0.4357$

$T_{L}=0.5089$

Soil albedo $=0.15$

No hot-spot

Planophile -

Uniform

Erectophile Uniform to make is as a function of the cosine of the solar zenith angle for two LAI's -1 and 8 - in Figs. $3 a-d$ for two surface hemispheric reflectances in the NIR -0.15 and 0.35 - and two in the PAR on 0.075 and 0.20 .

It is seen from the figures that the planophile LAND hemispheric reflectance always appears to be considerably different from that of the uniform and erectophile cases. The latter two are very alike except for small SZAs, similarly to what was found previously and validated by Monte-Carlo simulations (Dickinson et al. 1990; Pinty et al. 1990) for infinite optical depths. Second, the case of small LAIs is interesting for the erectophile canopy. For very large SZAs, when the underlying surface is not well illuminated, the hemispheric reflectance decreases with decreasing solar zenith angle as the sides of the leaves are less illuminated. When the sun rises higher in the sky, the surface underneath the canopy becomes more and more illuminated and the relative contribution of the leaves to the hemispheric reflectance decreases. For a surface hemispheric reflectance of 0.075 in the PAR band-Fig. $3 a$ - the decrease of the hemispheric reflectance with rising sun decreases rapidly and reaches an asymptotic value of 0.0365 already for a solar zenith angle of $60^{\circ}$. For the case of a surface hemispheric reflectance of 0.20 in the PAR band, the decrease of the hemispheric reflectance with rising sun is stopped and reversed and values over 0.07 are reached. The same general type of behavior is shown for the uniform case and even for the planophile case for a low LAI of 1 . This result confirms once more how important the interplay between the effects of the canopy structure and of the surface hemispheric reflectance may be.

Third, the deviations of the planophile from the uniform case are generally largest for small solar zenith angles, similarly to what was found, using different methods, by others for large optical depths (Dickinson et al. 1990). 

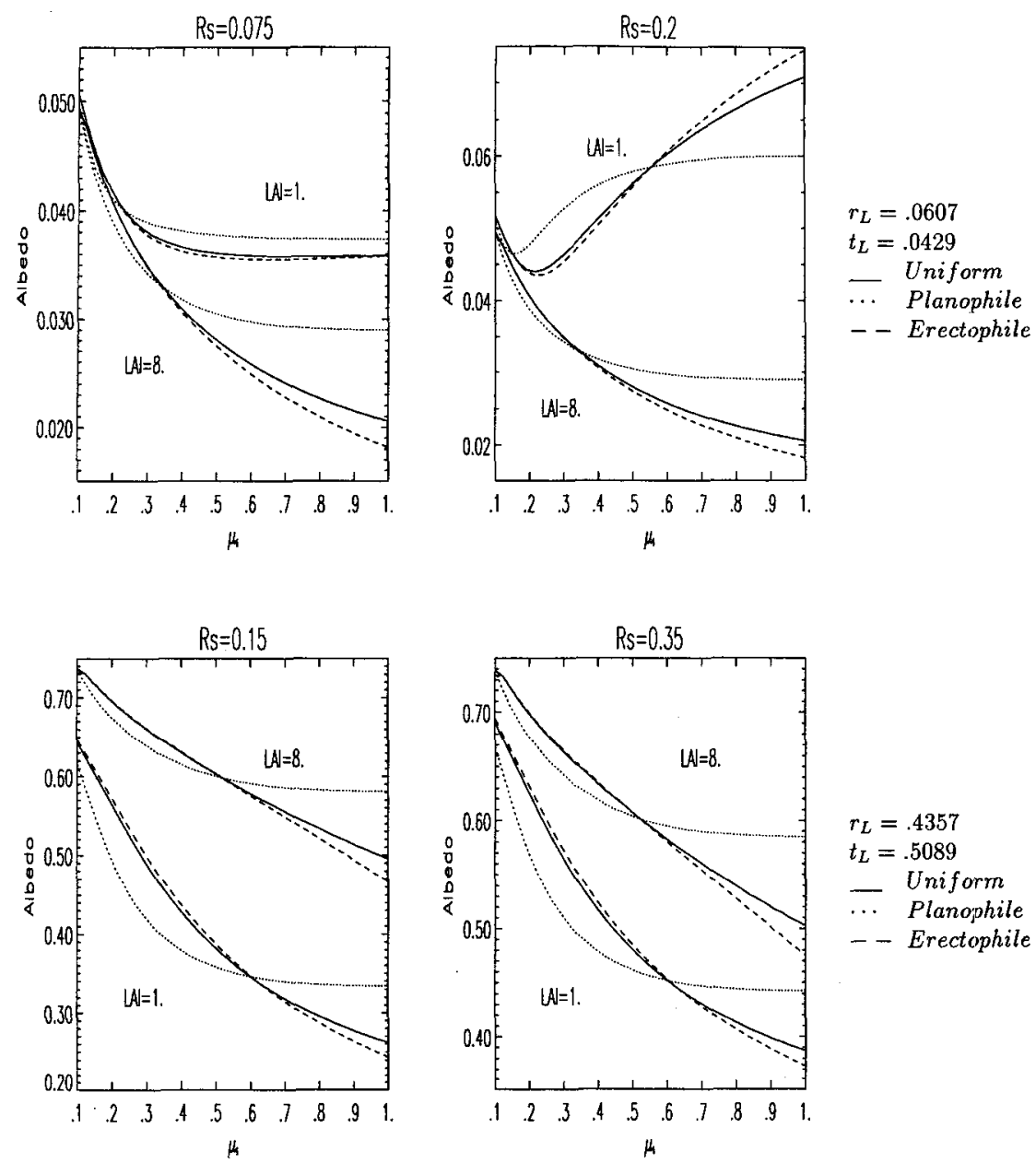

$$
\begin{aligned}
& r_{L}=.4357 \\
& t_{L}=.5089 \\
& -\quad \text { Uniform } \\
& \ldots \quad \text { Planophile } \\
& -- \text { Erectophile }
\end{aligned}
$$

Fig. 3. (a) Variation of hemispheric reflectance with $\cos (\mathrm{SZA})$ in the PAR band for surface hemispheric reflectance of 0.075 . LAI $=1$ and $8 ; r_{L}=0.0607, t_{L}=0.0429$. (b) Same as in Fig. $3 a$ for surface hemispheric reflectance of 0.2 . (c) Variation of hemispheric reflectance with $\cos (\mathrm{SZA})$ in the NIR band for surface hemispheric reflectance of 0.15 . LAI $=1$ and $8 ; r_{L}$ $=0.4357, t_{L}=0.5089$. (d) Same as in Fig. $3 \mathrm{c}$ for surface hemispheric reflectance of 0.35 .

In Figs. 4a-d we summarize the same data as a function of LAI, with LAND as a parameter in each figure for all surface hemispheric reflectances and low and high solar zenith angles in the NIR. In Figs. 5a-d, the same information is shown, but for the PAR band. It is clear from these two figures that

1) the hemispheric reflectance of the planophile case is systematically and significantly different from that of the erectophile and uniform LANDs

2) the deviations of the planophile case's hemispheric reflectances from those of the uniform one are almost independent of the surface hemispheric reflectance. This is because in the planophile case, the surface is completely covered by a canopy of horizontally oriented leaves for leaf area indices larger than one.
3) The deviation of the theoretical erectophile solution from that for the uniform one is small and systematic, largest for the small zenith angles.

For effective optical properties deduced from measurements of real vegetation, other factors, like the gap frequency distribution would also influence the values of the hemispheric reflectance.

Part of our results for large leaf area indices are validated by Monte Carlo ray-tracing simulations of hemispheric reflectance for canopies with isotropically scattering leaves (Dickinson et al. 1990; Pinty et al. 1990).

In our opinion, several practical conclusions may be drawn from these results. First, it is possible to use either of two LANDs - the uniform and the planophile ones - to model the distribution of leaves in vegetation for the purpose of modeling irradiances. 

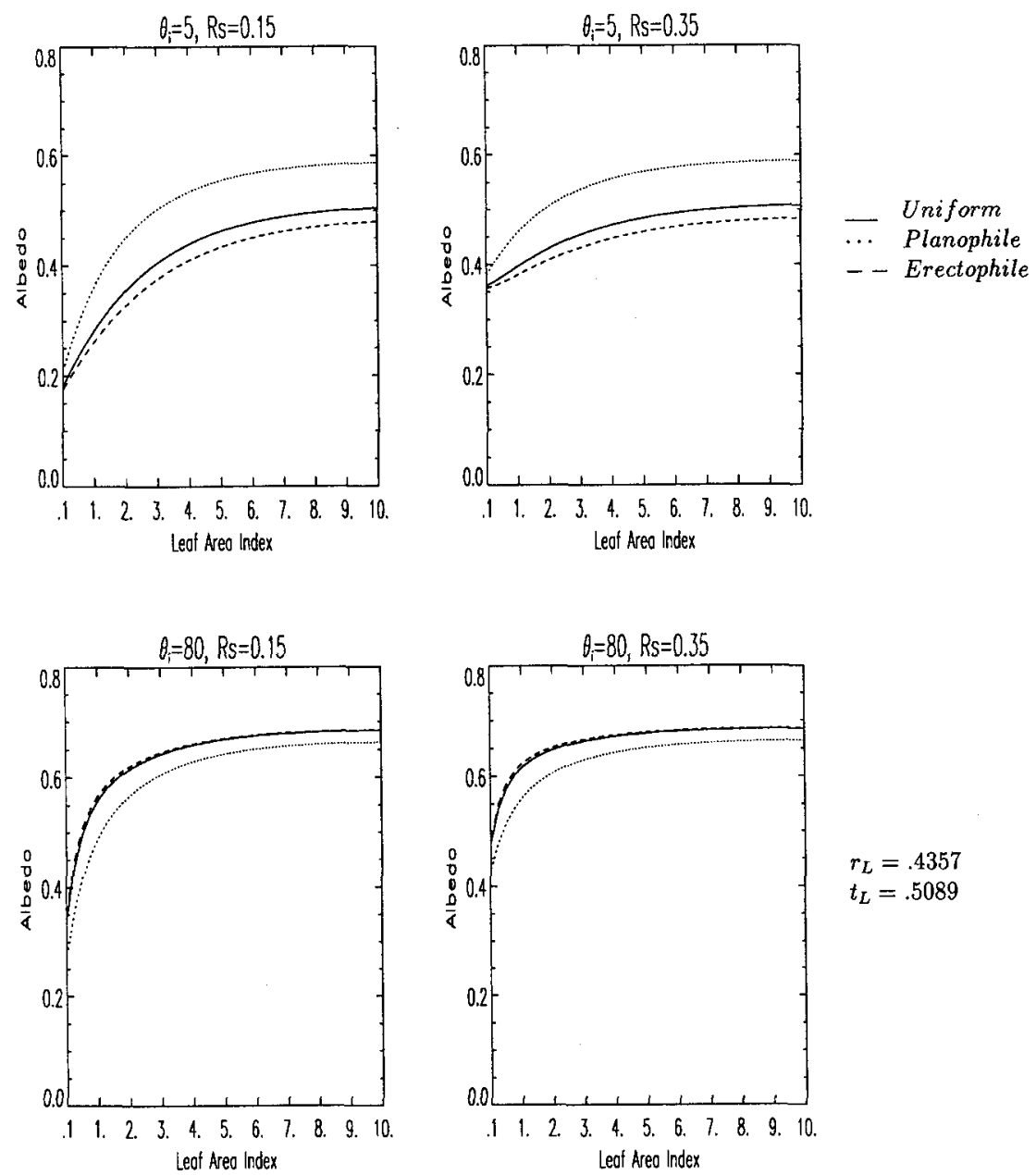

$$
\begin{aligned}
& r_{L}=.4357 \\
& t_{L}=.5089
\end{aligned}
$$

FIG. 4. (a) Comparison of variation of hemispheric reflectance with LAI for different LANDs in the NIR band. Surface hemispheric reflectance of $0.15 ; \mathrm{SZA}=5^{\circ} ; r_{L}=0.4357, t_{L}=0.5089$. (b) Same as in Fig. 4a for surface hemispheric reflectance of 0.35 . (c) Comparison of variation of hemispheric reflectance with LAI for different LANDs in the NIR band. Surface hemispheric reflectance of $0.15 ; \mathrm{SZA}=80^{\circ} ; r_{L}=0.4357, t_{L}=0.5089$. (d) Same as in Fig. $4 \mathrm{c}$ for surface hemispheric reflectance of 0.35 .

For the purpose of designing the radiative parameterization of vegetation, one should divide plants into two main classes - uniform and planophile. One could then treat the existence of the two classes of vegetation by having two separate cases in the analytical solution for the radiative transfer equation, the "planophile" case and the "uniform" one.

Such an algorithm would be easy to implement in any numerical model of the earth-atmosphere system. It would need as input the same three parameters as the other layers of the radiative system - the "effective" optical depth, albedo for single scattering, and asymmetry factor for each layer of vegetation.

These results of this section illustrate why appropriately chosen two-stream approximations should work fairly well for modeling the irradiances through veg- etation. The very heavy smoothing of the BRFs reduces the sensitivity of the irradiance to the exact shape of the BRF. This smoothing is strongest in those regions of zenith and azimuth angles where the BRFs for different LANDs are most variable and most different.

\section{Summary and discussion}

Remote sensing of plant properties requires the detailed analysis of radiances, $I\left(\mu, \mu^{\prime}, \phi, \phi^{\prime} ; z\right)$ (e.g., Ross 1981), which depend crucially and sensitively on both the exact architecture of the stand, the canopy, and the leaves as well as on the leaf spectral optical properties at the time of the measurement. It is therefore not justifiable to make an approximation like the twostream one for the radiances. On the other hand, radiation balances, heating rates, and photosynthesis 

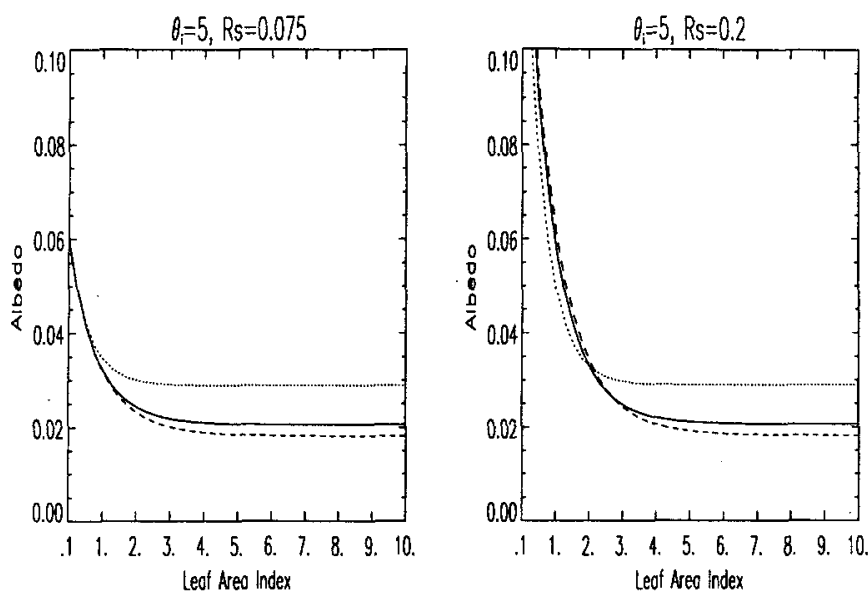

$$
\begin{aligned}
& \text { Uniform } \\
& \text {-. Planophile } \\
& \text { - Erectophile }
\end{aligned}
$$
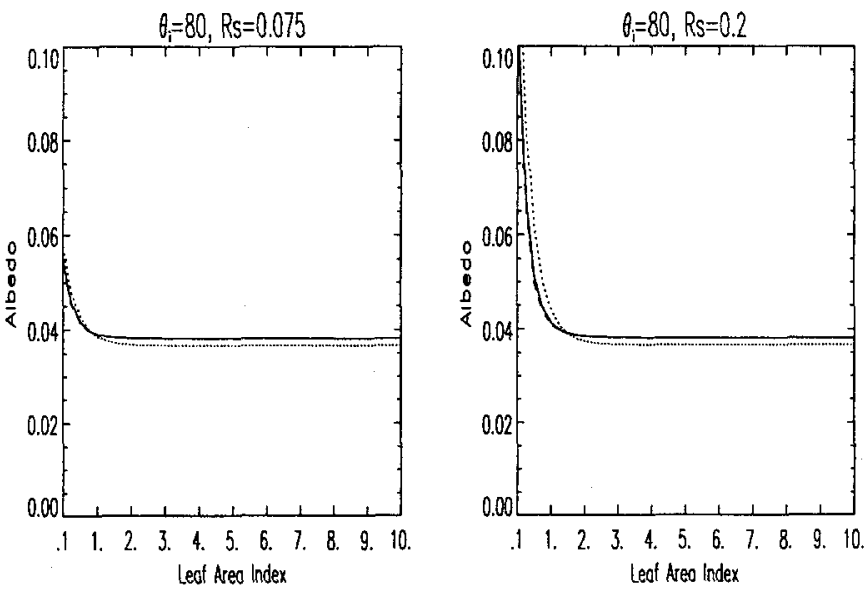

$r_{L}=.0607$

$t_{L}=.0429$

FIG. 5. (a) Comparison of variation of hemispheric reflectance with LAI for different LANDs in the PAR band for surface hemispheric reflectance of 0.075 and SZA $=5^{\circ} ; r_{L}=0.0607, t_{L}$ $=0.0429$. (b) Same as in Fig. 5a for surface hemispheric reflectance of 0.2. (c) Comparison of variation of hemispheric reflectance with LAI for different LANDs in the PAR band for surface hemispheric reflectance of 0.075 and $\mathrm{SZA}=80^{\circ} ; r_{L}=0.0607, t_{L}=0.0429$. (d) Same as in Fig. $5 c$ for surface hemispheric reflectance of 0.2 .

are induced by radiative energy fluxes - irradiances. These latter quantities are, by definition, weighted averages of the radiance field over the azimuth and zenith angle at a given location and time. The cosine of the zenith angle of emergence, $\mu$, is the weight for the intensity, $I\left(\mu, \phi ; \mu_{0}, \phi_{0}\right)$ (which is proportional to the BRF).

In this paper, we present a detailed test of the sensitivity of the flux RTE to the LAND. We have taken from the literature experimentally determined Ross functions of 17 plants and shown that their behavior as a function of viewing angle falls within a range outlined by those of the planophile and erectophile LANDs. We have demonstrated that the differences between irradiances based on a set of theoretical LANDs is very much reduced compared to those of the radiances. The rms error of the determination of hemispheric reflec- tance by remote sensing techniques is $\pm 10 \%$ (Pinty et al. 1990). We show, inter alia, that the hemispheric reflectances due to canopies with erectophile and uniform LANDs are similar to one another to within the experimental rms error and considerably different from that for the planophile case.

The use of either a uniform or a planophile distribution of leaf normals, together with the assumption of bi-Lambertian leaves with uniformly distributed azimuthal orientations, permits not only the reduction of the RTE to a conventional plane parallel form - as shown before-but also allows the use of available analytical two-stream solutions to the RTE (Ross 1981; Myneni et al. 1989; Joseph 1995).

It must be pointed out here that ad hoc use of twostream approximations have frequently made it possible in the past to model the hemispheric reflectances of 
vegetation canopies remarkably well - within the error of experimental data (e.g., Schwartzbart and Shaviv 1977; Dickinson et al. 1987, 1990). In a second paper, we shall show the same for both upward as well as downward irradiances through soybean and orange grove canopies, using the delta-Eddington two-stream approximation (Joseph et al. 1976).

The very important problem remains now of deciding which of the two possible solutions - uniform or planophile - to choose for a given type of plant or horizontally averaged set of plants at a given stage in its life cycle. We showed that the Ross functions of a set of 16 plants, derived from experimental data, all fall within the range of values defined by those for planoand erectophile LANDs and that it is possible to roughly divide the dataset into two groups.

The problem of how to classify vegetation in general into the two classes defined above should be approached through an extensive experimental study. The BRFs and all other parameters relevant for the description of vegetation of a large number of carefully chosen stands of plants or of suitable mixtures of plants should be assembled for various stages in their life and diurnal cycles and for various degrees of cover. Most methods of measurement lump together not only the leaves' physical and optical properties and those of the latters' distribution in orientation in the single canopy but also those of the trunk and branches, for example, the canopy gap frequency distribution and the relationships between the canopies of neighboring plants. The radiative properties derived from such measurements by use of a physical model are thus effective ones (Dickinson et al. 1990). These are, however, just the ones needed to translate the combined effects of many factors into a set of parameters acceptable to irradiation models used in numerical simulations of the atmosphere. The relevant parameters for each layer of vegetation need to be translated into those for use in twostream approximations - the optical depth, the albedo for single scattering, and some measure of the scattering phase function. The equivalent parameters for the different versions of two-stream approximations can be transformed one to the other (e.g., Meador and Weaver 1980). The possibility of deriving the parameters used in a delta-Eddington approximation from experimental data for soybean and orange grove canopies is demonstrated in another paper.

The largest differences between the BRFs of different types of plants occur mostly at large solar zenith angles and depend on azimuth. These differences are also those most important for the distinction between those plants by methods of remote sensing. It is exactly these features that are suppressed by the process of irradiance formation. It is therefore reasonable to assume that the irradiances through different types of vegetation will be very much more similar than the radiances and insensitive to many features used for remote sensing of vegetation features. This is one of the main points of this paper and augurs well for the future inclusion of vegetation as a set of fully coupled layers between the surface and the atmosphere in a radiation balance model.

Acknowledgments. The efficient and friendly hospitality and dynamic dedication to science at LAMP experienced during a sabbatical leave is gratefully acknowledged by one of us $(\mathrm{JHJ})$. Moreover, the detailed reviews of the anonymous referees contributed significantly to the quality of this paper.

\section{REFERENCES}

Avissar, R., 1992: Conceptual aspects of a statistical dynamical approach to represent landscape sub-grid scale heterogeneities in atmospheric models. J. Geophys. Res., 97, 2729-2742.

Baldocchi, D. D., B. A. Hutchinson, R. Matt, and R. T. McMillen, 1985: Canopy radiative transfer models for spherical and known leaf inclination angle distributions: A test in an oak-hickory forest. J. Appl. Ecol., 22, 539-555.

Bunnik, N. J. J., 1978: The multi-spectral reflectance of shortwave radiation by agricultural crops in relation with their morphological and optical properties. Mededelingen Landbouwhogeschool, Wageningen, The Netherlands.

Cohen, S., and M. Fuchs, 1987: The distribution of leaf area, radiation, photosynthesis and transpiration in a Shamouti orange hedgerow orchard. Agric. Forest Meteor., 40, 132-144.

Dickinson, R. E., P. J. Sellers, and D. S. Kimes, 1987: Hemispheric reflectances of homogeneous semi-infinite canopies: Comparison of two-stream analytic and numerical solutions. J. Geophys. Res., 92, 4282-4286.

_- B. Pinty, and M. M. Verstraete, 1990: Relating surface hemispheric reflectances in GCM to remotely sensed data. Agric. Forest Meteor., 52, 109-131.

Gutman, G., G. Ohring, and J. H. Joseph, 1984: The interaction between the geobotanic state and the climate-A modeling approach. J. Atmos. Sci., 41, 2663-2678.

Gutschick, V. P., and F. W. Wiegel, 1984: Radiation transfer in vegetation canopies and other layered media: Rapidly solvable exact integral equation not requiring Fourier resolution. J. Quant. Spectros. Radiat. Transfer, 31, 71-82.

Iaquinta, J., and B. Pinty, 1994: Adaptation of a bidirectional reflectance model including the hot spot to an optically thin canopy. (Physical Measurements and Signatures in Remote Sensing), ISPRS, Val d'IseÁre, France,

Joseph, J. H., W. Wiscombe, and J. A. Weinman, 1976: The deltaEddington approximation for radiative flux transfer. J. Atmos. Sci., 33, 2452-2459.

Kimes, D. S., 1984: Modeling the directional reflectance from complete homogeneous canopies with various leaf orientation distributions. J. Opt. Soc. of Amer. A, 1, 725-737.

- P. Sellers, and W. Newcomb, 1987: Hemispherical reflectance variations of vegetation canopies and implications for global and regional energy budget studies. J. Climate Appl. Meteor., 26, 959-972.

Knyazikhin, Y., and A. Marshak, 1991: Fundamental equations of radiative transfer in leaf canopies and itcrative methods for their solution. Photon-Vegetation Interactions, R. B. Myneni and J. Ross, Eds., Springer Verlag, 9-44.

, - and R. B. Myneni, 1991: Interaction of photons in a canopy of finite dimensional leaves. Remote Sens. Environ.

Koster, R. D., and M. J. Suarez, 1992: A comparative analysis of two land surface heterogeneity representations. J. Climate, 5, 13791390.

Kubelka, V. P., and F. Munk, 1931: Ein Beitrag zur Optik der Farbanstriche. Z. Tech. Phys., 11, 593-601.

Meador, W. E., and W. R. Weaver, 1980: Two-stream approximations to radiative transfer in planetary atmospheres: A unified 
description of existing methods and a new improvement. J. Atmos. Sci., 37, 630-643.

Myneni, R. B., G. Asrar, and E. T. Kanemasu, 1988: The theory of photon transport in leaf canopies. The Theory and Application of Optical Remote Sensing, G. Asrar, Ed., Wiley and Sons, 142-205.

$\longrightarrow, \ldots$, and -1989 : The theory of photon transport in leaf canopies. Theory and Applications of Optical Remote Sensing, G. Asrar, Ed., Wiley and Sons, 167-265.

—, A. Marshak, Y. Knyazikhin, and G. Asrar, 1991: Discrete ordinates method for photon transport in leaf canopies. PhotonVegetation Interactions, J. Ross and R. B. Myneni, Eds., Springer-Verlag, 45-110.

_-, G. Asrar, and F. G. Hall, 1992: A three-dimensional radiative transfer method for optical remote sensing of vegetated land surfaces. Remote Sens. Environ., 41, 105-121.

Pinty, B., M. Verstraete, and R. Dickinson, 1990: A physical model of the bidirectional reflectance of vegetation canopies. Part II: Inversion and validation. J. Geophys. Res., 95, $11767-11775$.

Ross, J., 1981: The Radiation Regime and Architecture of Plant Stands. 1st ed., W. Junk.
- - and T. Nilson, 1968: The spatial orientation of leaves in crop stands. Photosynthesis of Productive Systems, A. A. Nichprovich, Ed., Israel Prog. Sci. Trans., 86-99.

Schwartzbart, I., and G. Shaviv, 1976: A Monte-Carlo model for the radiation field in plant canopies. Agric. Meteor., 17, $333-$ 353.

Sellers, P. J., Y. Mintz, Y. C. Sud, and A. Dalcher, 1986: A simple biosphere model $(\mathrm{SiB})$ for use within general circulation models. J. Atmos. Sci., 43, 505-531.

Shettle, E. P., and J. A. Weinman, 1970: The transfer of solar irradiance through inhomogeneous turbid atmospheres evaluated by Eddington's approximation. J. Atmos. Sci., 27, 1048-1055.

Shifrin, K. S., 1968: Scattering of light in a turbid medium. NASA Tech. Rep. TT F-477, 212 pp.

Tucker, C. J., 1979: Red and photographic infra-red linear combination for monitoring vegetation. Remote Sens. Environ., 8, $127-150$.

Verstraete, M. M., 1987: Radiation transfer in plant canopies: Transmission of direct solar radiation and the role of leaf orientation. J. Geophys. Res., 92, $10985-10995$. 